\title{
Fragmentation in Nocardia corallina
}

\author{
By O. BROWN* AND J. B. CLARK \\ Department of Botany and Microbiology, University of Oklahoma, \\ Norman, Oklahoma, U.S.A.
}

(Received 23 May 1966)

\begin{abstract}
SUMMARY
The growth cycle of Nocardia corallina involves a period of coenocytic hyphal development terminated by fragmentation. Hyphal elongation occurred by insertion of new material at the hyphal tips, not by intercalary growth. Exceptions occurred at points of branch initiation. Development of cross-walls preceded fragmentation and the new tips so produced were also capable of elongation. The fragmentation process appeared to be initiated by agents which accumulated in the medium, since used broth from actively fragmenting cultures stimulated earlier fragmentation in assay inocula. This effect was not shown by media from cultures in the coenocytic hyphal phase, nor by old resting cultures. The population density also affected fragmentation in a manner which suggested that accumulation of threshold concentrations of a diffusible metabolic product was required. A Millipore filter technique for assaying the effects of agents involved in fragmentation is described.
\end{abstract}

\section{INTRODUCTION}

Members of the genus Nocardia have similar growth cycles which involve periods of hyphal development terminated by fragmentation. The extent and duration of hyphal development vary with environmental conditions, but have been used as criteria for delineation of the genus as well as for speciation (McClung, 1949, 1954). The growth cycles of several species have been carefully described, but the mechanisms which control morphogenesis are unknown. However, fragmentation in Nocardia corallina appears to be induced by agents which accumulate in the growth medium (Webb \& Clark, 1957). A further study of this phenomenon is reported here, dealing specifically with the fragmentation sequence, additional evidence for metabolic factors involved in fragmentation, and a method of assaying such factors.

\section{METHODS}

Organism. Nocardia corallina ATCC 4273 was maintained on nutrient agar $+0.5 \%$ fructose. Cultures were transferred at $48-\mathrm{hr}$ intervals (a time sufficient for completion of the growth cycle) to provide reproducible inocula for all experiments.

Observation of growing cultures. Agar block cultures were grown at $25^{\circ}-26^{\circ}$ under coverslips on hanging-drop slides by using procedures similar to those described by McClung (1949). The agar blocks were cut from nutrient agar containing $0.5 \%$ glucose, and the coverslips were sealed only on two sides and positioned off-centre

* Present address: Department of Microbiology, School of Medicine, University of Missouri, Columbia, Missouri. U.S.A. 
to provide an opening for diffusion of air into the chamber. The development of individual organisms was microscopically observed and photographed at intervals.

Photomicrography. Photomicrographs were taken on Kodak High Contrast $35 \mathrm{~mm}$. film with an apochromatic lens system (limiting n.a., 1·3). Living organisms were photographed with the substage condenser diaphragm almost closed to give maximum contrast and to expose the organisms to a minimum of light. Phase microscopy was attempted, but a higher light intensity was then required and the organisms did not germinate normally.

Fragmentation studies. Growth on solid medium was measured by using a series of Millipore filters inoculated with equal numbers of organisms and incubated at $29^{\circ}$ on the surface of semi-solid nutrient agar $(0.75 \%$ agar $)$ containing $0.5 \%$ fructose. The Millipore filters were removed after various intervals of incubation and placed in screw-cap tubes containing $0.9 \% \mathrm{NaCl}$ solution and large beads. The organisms were freed from the filter and evenly suspended by mixing first with the Vortex Jr. mixer (Scientific Industries, Inc., Queens Village, N.Y.) and then with the Disontegrator (Ultrasonic Industries, Inc., Albertson, L.I., N.Y.). Viable units were determined by standard plate colony-counting techniques. Slides were made at intervals and stained by the Chance nuclear stain (Chance, 1952) or the Webb cell-wall stain (Webb, 1954).

Detection of fragmentation stimulation. Cultures were grown in nutrient broth + $0.5 \%$ fructose and aerated with sterile air under pressure. Samples of culture were removed after various intervals of incubation and freed from organisms by centrifugation and filtration; culture filtrates so prepared will hereafter be referred to as ' used media'. Preliminary assays were done by inoculating the used media as described by Webb \& Clark (1957). Several advantages were found for the following modification, which uses surface cultures rather than broth cultures for assay. The assay inoculum was prepared as follows. The growth from 48 -hr cultures was harvested from fructose agar and washed three times with $0.9 \% \mathrm{NaCl}$ solution by alternate suspension and centrifugation for $10 \mathrm{~min}$. at $10,000 \mathrm{~g}$ in a Servall refrigerated centrifuge at $4^{\circ}$. A standard curve, relating extinction and number of colony-forming units, was prepared for convenience in reproducing inoculum sizes. Equal samples were filtered through Millipore filters (HAWP $0 \cdot 45 \mu$ ), thus producing a series of filters inoculated with equal numbers of evenly distributed organisms. The filters were transferred to sterile Millipore pads, saturated with fresh fructose broth for control cultures, or with samples of used medium for determining fragmentation-stimulation activity. After intervals of incubation, slides were prepared and evaluated as previously described. An inoculum of $10^{7}$ organisms/filter with evaluation of slides after $6 \mathrm{hr}$ was found to be adequate for the assay.

\section{RESULTS}

\section{Fragmentation}

The fragmentation sequence of a developing microcolony is shown in Plates 1-3, figs. 1-13. Routinely, the initial cross-wall formed near the centre of the hypha (fig. 1). After fragmentation, elongation occurred from both the new tips. Comparisons of Pl. 1, figs. 1-3, show that elongation occurred only at the tips of the hyphae, including the new tips produced by fragmentation. Intercalary growth did not occur, except at 
the point of branch initiation (Pl. 1, fig. 4). This is more clearly shown in Fig. 1 which depicts results of measurements made with a filar micrometer on developing hyphae with multiple branches which served as markers. The average rate of elongation for the two ends of the original filament was the same, about $0.02 \mu / \mathrm{min}$. The average rate of increase for the newly developing branches was slightly less.

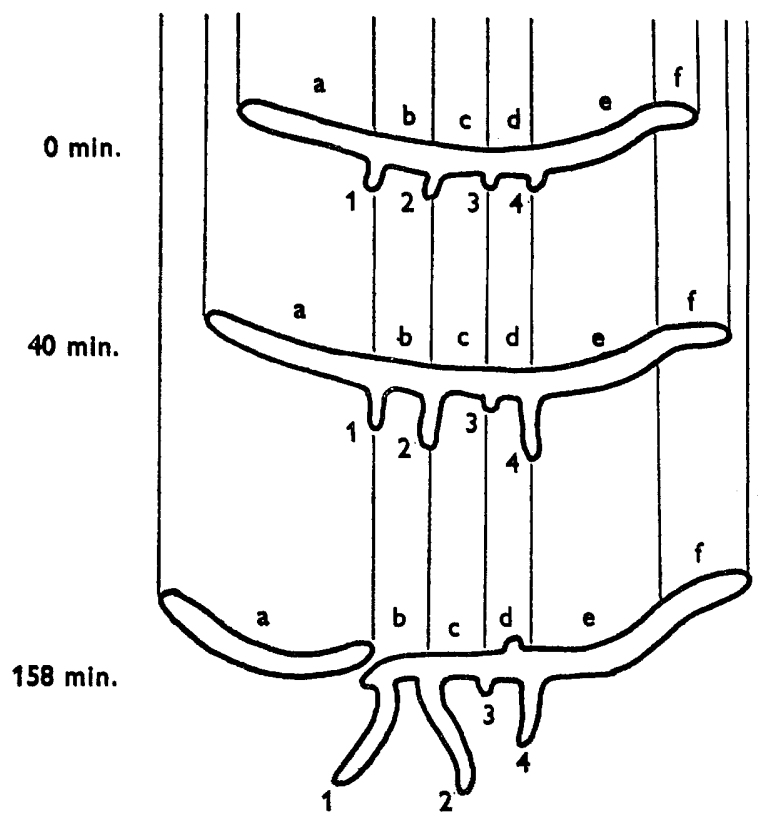

Fig. 1. Growth of hyphae of Nocardia corallina by elongation from the tips. The values listed below give the growth rates for the indicated portions of the hyphae shown in the figure :

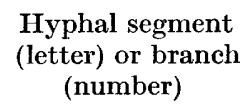

a
b
c
$\mathrm{d}$
$\mathrm{e}$
$\mathrm{f}$
$\mathbf{1}$
$\mathbf{2}$
$\mathbf{3}$
4

\begin{tabular}{ccc}
\multicolumn{3}{c}{ Length $(\mu)$ at } \\
\hline 0 min. & 4.0 min. & $158 \mathrm{~min}$. \\
5.34 & 6.10 & 8.45 \\
$2 \cdot 12$ & 2.07 & 2.09 \\
$1 \cdot 91$ & $2 \cdot 38$ & 2.02 \\
1.69 & 1.45 & 1.48 \\
3.39 & 3.49 & 3.37 \\
1.57 & 2.03 & 4.67 \\
1.67 & 2.16 & 4.30 \\
1.73 & 2.50 & 4.52 \\
0.57 & 0.71 & 0.57 \\
0.89 & 1.01 & 2.92
\end{tabular}

Total change in

$$
\begin{array}{r}
+3 \cdot 11 \\
-0.03 \\
+0.11 \\
-0.21 \\
-0.02 \\
+3 \cdot 10 \\
+2.63 \\
+2.79 \\
0.00 \\
+2.03
\end{array}
$$

In Pl. 1, fig. 5, and Pl. 2, fig. 6, the terminal portions of the original hyphae are still coenocytic and elongating. In Plate-figs. 7-10 (Pls. 2, 3), a wave of cross-wall formation has progressed outward from the middle, producing short fragments of approximately equal length. Elongation had essentially ceased in figs. 11-13 (Pl. 3), but cross-walls continued to be formed and a uniform population of short fragments was produced; in fig. 13 some coccoid elements are to be seen. 
Fragmentation stimulation by cell-free culture filtrates

Fragmentation was not stimulated by medium from 1-day cultures nor by medium from week-old growth. Used media from 2-, 3- and 4-day cultures showed progressively greater fragmentation-stimulation of assay inocula. Cytologically normal germination occurred in all cases, but the coenocytic hyphal phase was shortened because of earlier and more extensive formation of cross-walls. With 3- and 4-day used media, cross-wall formation began after only a few nuclear divisions, while the hyphae were still very short. The typical development of long coenocytic hyphae as seen in control cultures was not observed. The fragmentation stimulation effects of used media were not destroyed by boiling for $5 \mathrm{~min}$.

Figure 14 depicts typical results obtained by the Millipore filter assay technique. The control (Fig. 14A) supplied with fresh nutrient broth is typical of a $6 \mathrm{hr}$ culture under these conditions. Fig. 14B shows a culture grown for the same period in used media taken from a 3-day fragmenting culture. Most of the organisms contain multiple cross-walls and some are fragmenting.

Table 1. Effects of inoculum size upon fragmentation in cultures of Nocardia corallina grown on the surface of Millipore filters

\begin{tabular}{ccc}
$\begin{array}{c}\text { Total no. } \\
\text { organisms } \\
\text { inoculated/filter }\end{array}$ & $\begin{array}{c}\text { Organisms inoculated/ } \\
\mu^{2} \text { surface area } \\
\text { of medium }\end{array}$ & \multicolumn{1}{c}{$\begin{array}{c}\text { Onset of fragmentation } \\
(\mathrm{hr})\end{array}$} \\
$4 \times 10^{5}$ & $0 \cdot 0042$ & 22 \\
$4 \times 10^{6}$ & $0 \cdot 042$ & 14 \\
$4 \times 10^{7}$ & $0 \cdot 42$ & $12-14$ \\
$4 \times 10^{8}$ & $4 \cdot 2$ & $\begin{array}{l}\text { No coenocytic hyphal phase; } \\
\text { initial growth with cross-wall } \\
\end{array}$ \\
& & formation
\end{tabular}

Table 2. Growth rates of Nocardia corallina on Millipore filters

$\begin{array}{ccc}\begin{array}{c}\text { Incubation time } \\ (\mathrm{hr})\end{array} & \begin{array}{c}\text { Extinction at } \\ 475 \mathrm{~m} \mu^{*}\end{array} & \begin{array}{c}\text { Organisms (colony } \\ \text { count)/filter } \\ \left(\times 10^{-8}\right)\end{array} \\ 0 \dagger & 0 \cdot 035 & 4 \cdot 3 \\ 0 & - & 4 \cdot 1 \\ 5 \cdot 5 & 0 \cdot 128 & 6 \cdot 5 \\ 8 \cdot 25 & 0 \cdot 205 & 13 \cdot 8 \\ 8 \cdot 8 & 0 \cdot 207 & 20 \cdot 9 \\ 20 \cdot 5 & 0 \cdot 581 & 54 \cdot 0 \\ 32.5 & 0.964 & 91 \cdot 6\end{array}$

* Total filter population resuspended in $10 \mathrm{ml}$. of $0.9 \% \mathrm{NaCl}$ solution.

$\dagger$ Figures in this row were obtained from a sample of the suspension subsequently used to inoculate the filters.

\section{Effects of population density upon fragmentation}

The duration of the coenocytic hyphal phase was also dependent upon the initial inoculum size; with larger inocula, fragmentation occurred sooner (Table 1). When the inoculum was increased to give more than a monolayer, e.g. $4 \cdot 2$ organisms $/ \mu^{2}$ surface, the typical coenocytic phase did not occur. Cytological evidence indicated that such organisms were growing by elongation and multiplying by cross-wall pro- 
duction at, essentially, each nuclear division. To gain further evidence about multiplication under these conditions, the growth rates of surface colonies were determined (Table 2). The inoculum, $4.3 \times 10^{8}$ organisms/Millipore filter, was sufficient to provide a layer several organisms thick. Under these conditions, proliferation did occur, but the normal phase of long coenocytic hyphae (as shown in Plate 1, fig. 1) did not occur. Instead, cross-walls were produced soon after germination and the culture had an appearance similar to that shown in Plate 4, fig. 14B.

The data in Table 2 also provide information about the increase in cell mass relative to proliferation by fragmentation. The extinction increased by a factor of $3 \cdot 6$ during the first $5 \cdot 5 \mathrm{hr}$, while the number of organisms (colony count) increased by 1.5 . This indicated an increase in size of organism and some multiplication, in agreement with the cytological observations. During the period, 5.5-8.25 hr, the extinction increased by a factor of $1 \cdot 6$ while the population (colony count) doubled. This also agreed with cytological evidence that elongation was accompanied by fragmentation. During the period, $8 \cdot 25-8 \cdot 8 \mathrm{hr}$, there was essentially no increase in extinction; however, there was an increase of $\mathbf{1 . 5}$ in numbers of organisms (colony counts), which indicated that some fragmentation occurred. During the period, $8 \cdot 8-$ $20.5 \mathrm{hr}$, the increases in extinction and in population were $2 \cdot 8$ and $2 \cdot 6$, respectively, indicating that fragmentation kept pace with synthesis of new cell mass. This consistent relationship continued through the $32.5 \mathrm{hr}$ observation with ratios near 1.7. For the total $32.5 \mathrm{hr}$ period, the extinction increased by a factor of 27.5 while the population increased by a factor of $\mathbf{2 2 \cdot 3}$.

\section{DISCUSSION}

Observations of growing Nocardia corallina indicated that insertion of new cellwall material, which accompanied growth and elongation, occurred at the tips but not along interior portions of hyphae. Exceptions occurred wherever cross-walls formed; the two new tips, produced then, served as loci for elongation. In addition, the origin of branches represented loci where new cell-wall material was inserted. At present it is not clear whether these three germinal areas share common mechanisms.

Since intercalary growth did not occur, and cross-septation initially occurred near the middle of a given hypha and progressed down the filament toward the tip, it follows that fragmentation occurred first in the older parts of the hypha. Since fragmentation resulted in production of more growing tips, there was a greater proliferation near the origin of a developing microcolony. It seems reasonable that this region is also the area where diffusible metabolic products would accumulate most rapidly. During the early stages of growth, the elongating tips, which remain free from cross-walls for the longest period, are being extended into a fresh environment and the pattern of fragmentation seen in figs. 1-13, Pls. 1-3, is consistent with what would be predicted if fragmentation were induced by a diffusible metabolic product.

The fragmentation-stimulation effect of used medium was correlated with growth cycle stages which actively formed cross-walls. Its absence from young cultures in coenocytic growth and week-old non-multiplying cultures suggests that the agent responsible is produced by the developing culture and subsequently used by the organisms or is decomposed because of inherent lability.

Germination to coenocytic hyphae is typically associated with the process of 
transfer which initiates a new culture. Among other changes which occur during such transfers, there is a great decrease in the number of organisms. If fragmentation were dependent upon some critical concentration of metabolic by-products, then transfer would remove the organisms from such products and the decrease in population would decrease their rate of re-accumulation. The coenocytic phase would then coincide with the time required for the fragmentation factors to accumulate to threshold concentration. If such were the case, the effects of culture transfer to new medium should be partially negated by transferring a mass inoculum, since a high population density should result in a more rapid accumulation of the metabolic factors involved. Tables 1 and 2 show that such an effect was obtained with mass inocula which were washed to remove most of the extracellular metabolic by-products. When these cultures were initiated with inocula containing sufficient organisms to cover the surface completely, the typical coenocytic hyphal phase did not occur and growth and multiplication occurred by cytokinesis accompanied by karyokinesis. This same type of growth and multiplication is typical of normal cultures only after the population has developed to a similar density through germination, coenocytic hyphal development and stages of accelerated cross-wall formation.

The Millipore filter assay technique offers several advantages for studying the fragmentation-stimulation effects of used media. The process is rapid, requiring only $6 \mathrm{hr}$ incubation, and the development of the growth cycle in control cultures is uniform, with fragmentation a more synchronous and more sharply defined event than that seen in broth cultures; hence the effects of used media are more clearly shown. The filter technique also allows accurate reproduction of population density and distribution. With the availability of this assay procedure it appears that further study of metabolic factors involved in morphogenesis of the nocardias will be feasible.

\section{REFERENCES}

Chance, H. L. (1952). Crystal violet as a nuclear stain for Gaffkya tetragena and other bacteria. Stain Technol. 27, 253.

McClung, N. M. (1949). Morphological studies in the genus Nocardia. I. Developmental studies. Lloydia 12, 137.

McClung, N. M. (1954). Morphological studies in the genus Nocardia. III. The morphology of young cultures. Ann. N.Y. Acad. Sci. 60, 169.

WEBB, R. B. (1954). A useful bacterial cell wall stain. J. Bact. 67, 252.

Webb, R. B. \& Crark, J. B. (1957). Cytogenetic study of Nocardia corallina. J. Bact. 74, 31.

\section{EXPLANATION OF PLATES}

\section{Plates 1-3}

Figs. 1-13. The fragmentation sequence in Nocardia corallina. Photographs were taken at the following time intervals: fig. 1, $12 \mathrm{hr}$; fig. $2,13 \mathrm{hr}, 50 \mathrm{~min}$.; fig. $3,15 \mathrm{hr}, 15 \mathrm{~min}$.; fig. 4, $16 \mathrm{hr}$, 55 min.; fig. 5, 18 hr, 30 min.; fig. 6, $20 \mathrm{hr}, 45 \mathrm{~min}$; f fig. 7, 24 hr, $45 \mathrm{~min}$.; fig. 8, $27 \mathrm{hr}, 10 \mathrm{~min}$. ; fig. 9, $28 \mathrm{hr}, 10 \mathrm{~min}$; f fig. 10, $30 \mathrm{hr}, 40 \mathrm{~min}$.; fig. 11, $32 \mathrm{hr}, 40 \mathrm{~min}$.; fig. 12, 34 hr, $40 \mathrm{~min}$.; fig. 13, $39 \mathrm{hr}, 40 \mathrm{~min} . \times 3500$.

Plate 4

Fig. 14. Stimulation of cross-wall formation by used medium. The control culture of Nocardia corallina (A) was grown on a Millipore filter placed on a pad saturated with fresh nutrient broth + $0.5 \%$ fructose. The test culture (B) was treated identically except that used broth from a 3-day culture was substituted as nutrient. Both filters were inoculated by filtration with $10^{7}$ organisms from the same suspension. Organisms were stained by the Webb cell-wall procedure (1954) and photographs were taken after incubation for $6 \mathrm{hr}$ at $29^{\circ} . \times 3000$. 


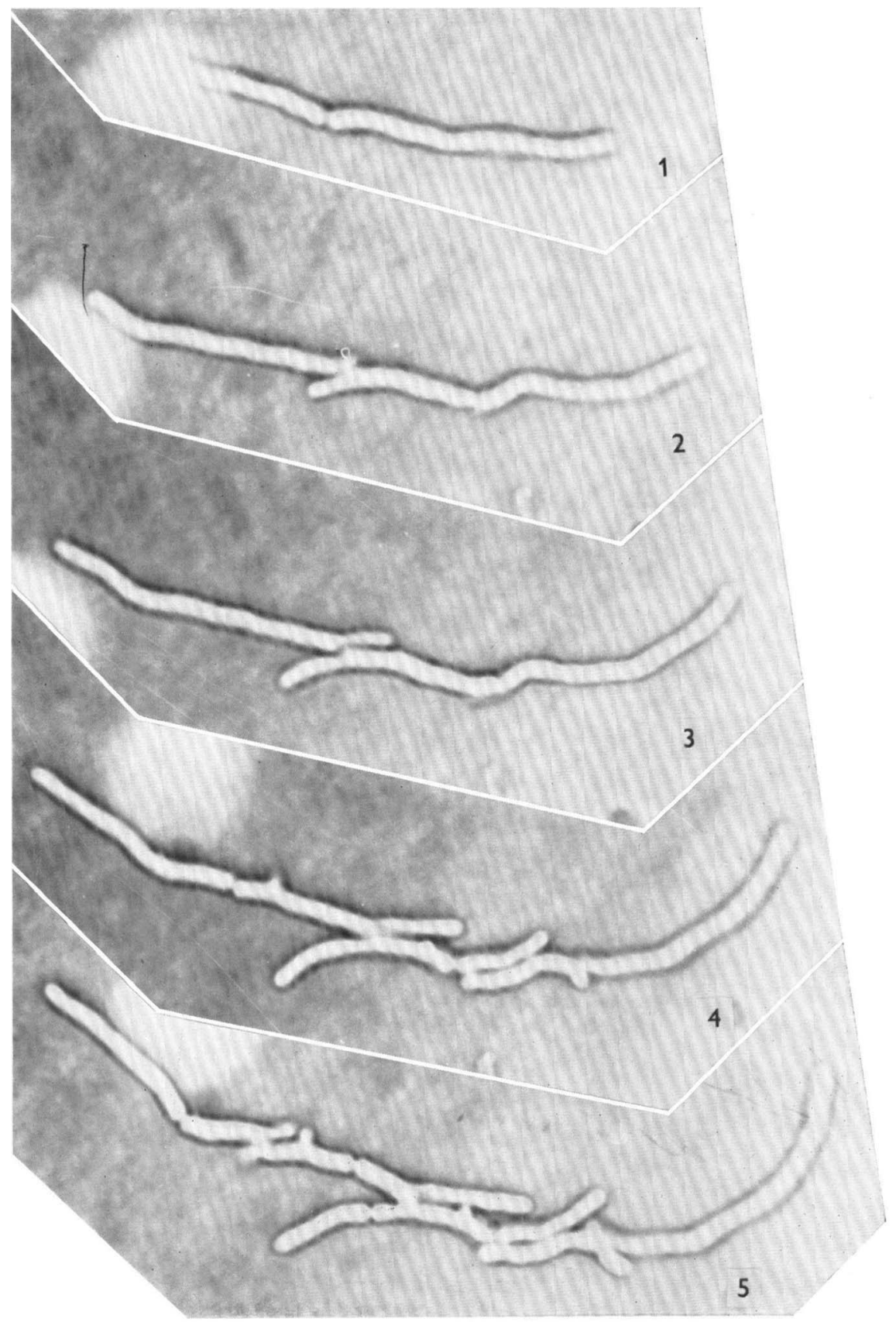




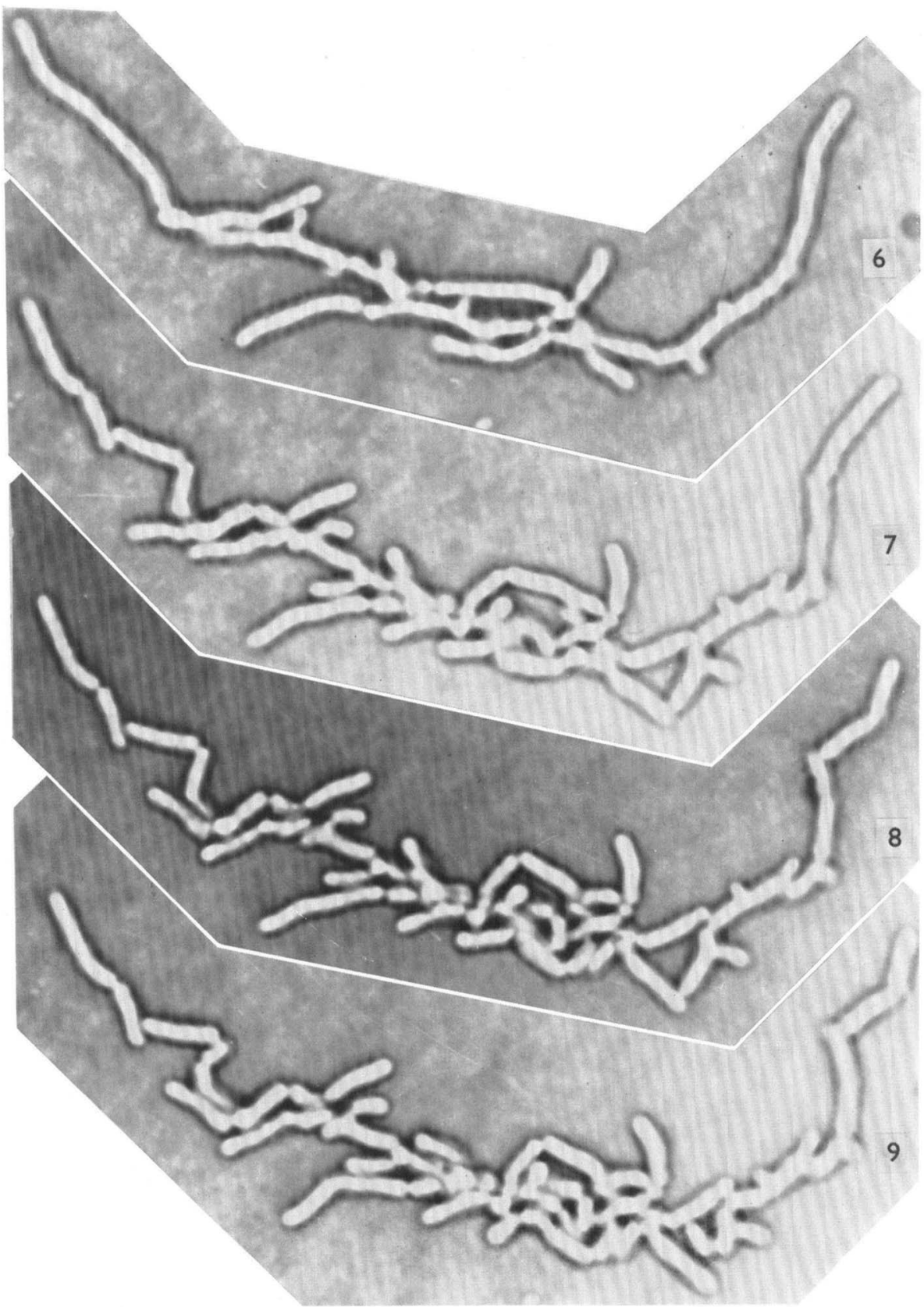

O. BROWN AND J. B. CLARK 


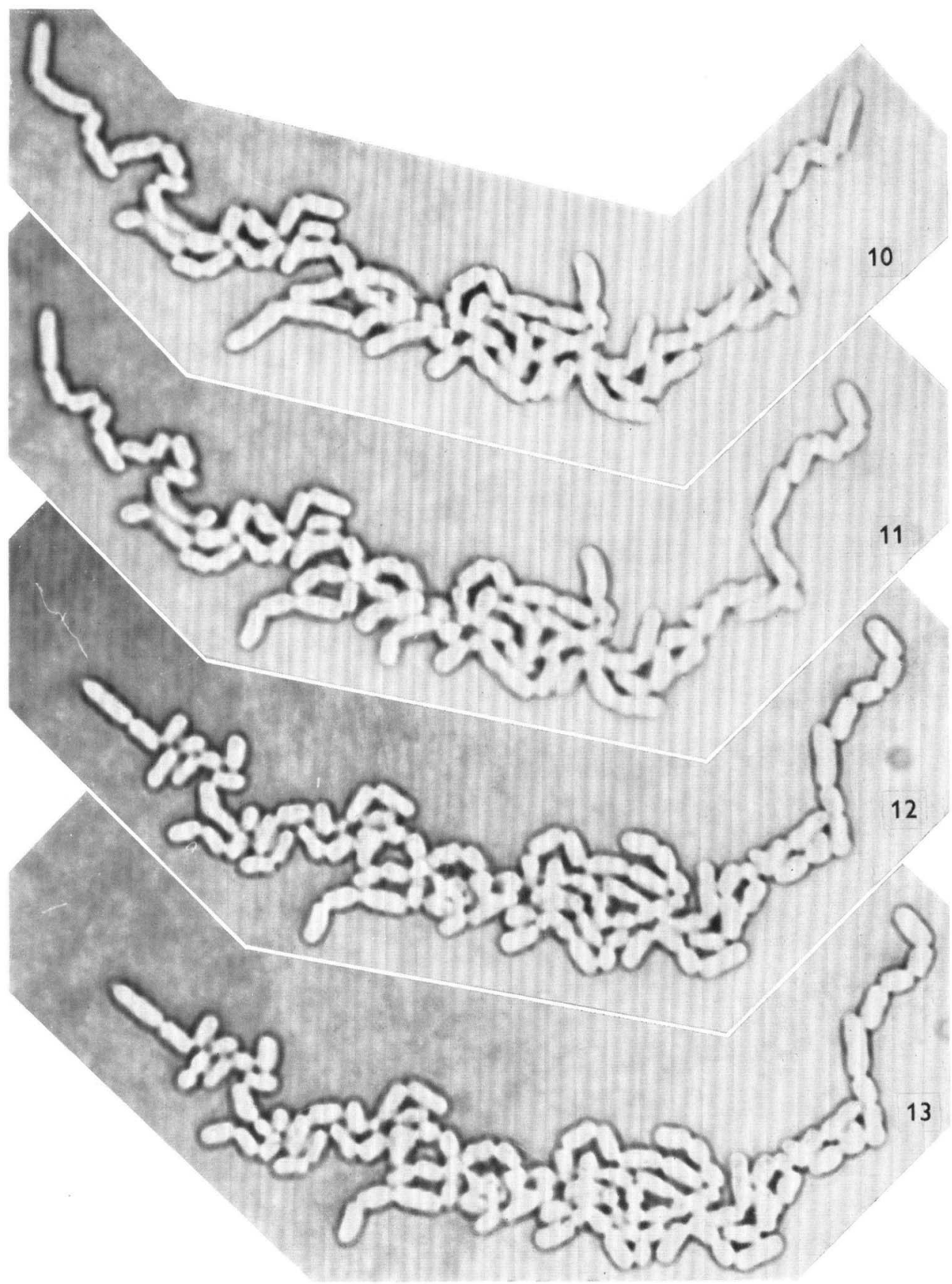

O. BROWN AXI J. B. CLARK 


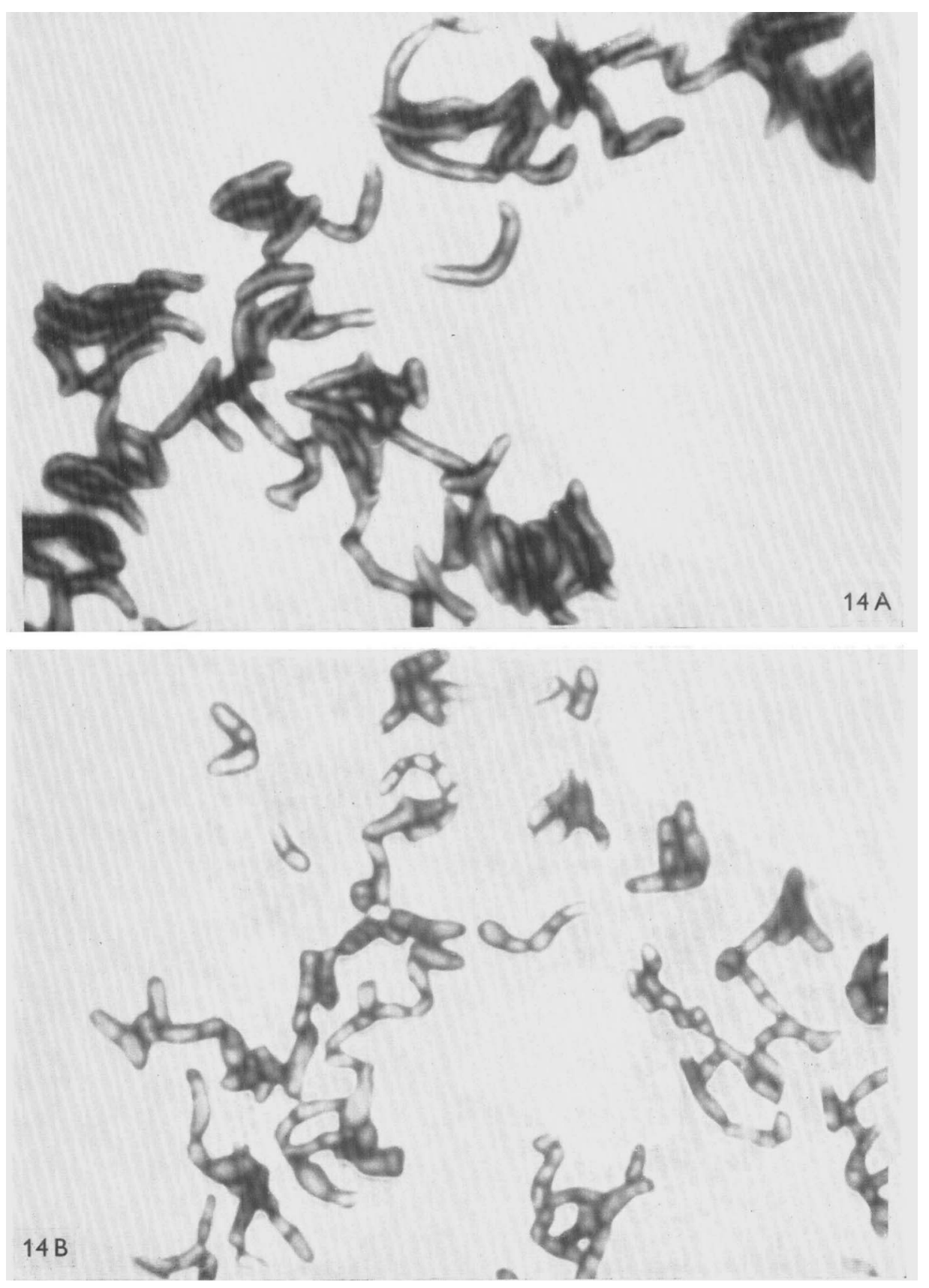

O. BROWN AND J. B. CLARK 